\title{
On martingales and the use of optional stopping theorem to determine the mean and variance of a stopping time
}

\author{
Ganiyu, A. A. ${ }^{1}$, Fakunle, I. $^{2}$ \\ Department of Mathematics, Adeyemi College of Education, Ondo, Nigeria \\ Email address: \\ ganiyuiwajowa@yahoo.com (Ganiyu, A. A.)
}

\section{To cite this article:}

Ganiyu, A. A., Fakunle, I.. On Martingales and the Use of Optional Stopping Theorem to Determine the Mean and Variance of a Stopping Time. Applied and Computational Mathematics. Special Issue: Computational Finance. Vol. 3, No. 6-1, 2014, pp. 12-17. doi: 10.11648/j.acm.s.2014030601.13

\begin{abstract}
This paper examines the roles martingale property played in the use of optional stopping theorem (OST). It also examines the implication of this property in the use of optional stopping theorem for the determination of mean and variance of a stopping time. A simple example relating to betting system of a gambler with limited amount of money has been provided. The analysis of the betting system showed that the gambler leaves with the same amount of money as when he started and therefore satisfied martingale property. Linearity of expectation property was used as a reliable tool in the use of the martingale property.
\end{abstract}

Keywords: Martingales, Gambler, Random Walk, Stopping Time, Optional Stopping Theorem, Mean, Variance

\section{Introduction}

In probability theory, a martingale is a model of a fair game where knowledge of the past events will never help to predict the future winnings. In particular, a martingale is a sequence of random variables (that is, a stochastic process) for which, at a particular time in the realized sequence, the expectation of the next value in the sequence is equal to the present observed value even given the knowledge of all prior observed value at a current time. The original meaning of martingale was stated by Kannan [1997] in Ugbebor, Ganiyu and Fakunle [2012] and Hazewinkel [2001]

An intuition about gambling as stated by Karlin and Taylor [1975 ] in Ugbebor, Ganiyu and Fakunle [2012] is that a gambler cannot turn a fair game into an advantageous one by periodically deciding to double the bet or by cleverly choosing the time to quit playing. This intuition invariably led to optional stopping theorem (OST).

There are various applications of martingales. For example Ugbebor and Ganiyu [2007] applied the martingale model to the NGN/USD exchange rate. OST has many applications. For example, it was applied in risk theory by Shiu and Gerber (1994a), Shiu, and Gerber (1994b), Shiu and Gerber (1996a), and Shiu and Gerber (1996b). The OST can also be applied to prove the impossibility of successful betting strategy of a gambler with a finite lifetime and a house limit on bet.

This paper examines the roles played by martingale property in the use of (OST). It highlights that martingale property is a condition that must be satisfied before the use of (OST). To see this claim, (OST) was used to determine the mean and variance of a stopping time. A simple example relating to betting system of a gambler with limited amount of money has also been provided. The analysis of the betting system showed that the gambler leaves with the same amount of money as when he started and therefore satisfied martingale property.

\section{Preliminaries}

Definition 2.1

Consider discrete random variable $X$ and $Y$. Let $S_{X}=\{x \mid P[X=x]>0\}$. The conditional expectation of $Y$ given that $X=x$ has occurred, where $x \in S_{X}$ is defined by

$$
E[Y \mid X](x)=E[Y \mid X=x]=\sum_{y} y P\{Y=y \mid X=x\}
$$




\section{Theorem 2.1}

Let $\mathrm{Y}$ be independent of $X$ and $E[Y \mid X]=\psi(Y)$. Then, the function $\psi(X)=E[Y \mid X]$ satisfies

$E[\psi(X)]=E[Y]$. (See reference no [Ganiyu (2006)] for the proof).

Definition 2.2

Consider random variables $X_{1}, \ldots, X_{n}$. Denote by $\mathrm{F}_{n}$ the $\sigma-$ algebra (i.e. collection of events) generated by these random variables which satisfies the properties (i) $\Omega \in \mathrm{F}_{n}$ (ii) $A \in \mathrm{F}_{n} \Rightarrow A^{c} \in \mathrm{F}_{n}$ and (iii) $A_{1}, \ldots, A_{k} \in \mathrm{F}_{n} \Rightarrow \bigcup_{n=1}^{\infty} A_{n} \in \mathrm{F}_{n}$, then

$$
\mathrm{F}_{n}=\sigma\left(X_{1}, \ldots, X_{n}\right), n \geq 0 .
$$

Theorem 2.2 (Linearity property of conditional expectation)

Let $Y, U$ and $V$ be discrete random variables. If the scalars $a, b \in \mathbb{R}$, then

$$
E[(a U+b V) \mid Y]=a E(U \mid Y)+b E(V \mid Y)
$$

(See [Ganiyu (2006)] for the proof).

\section{Definition 2.3}

A stochastic process $\left\{X_{n}, n \geq 0\right\}$ is said to be a martingale with respect to a process $\left(Y_{n}, n \geq 0\right)$, if for all

$$
n \geq 0, E\left[X_{n}\right]<\infty \text { and } E\left[X_{n+1} \mid Y_{0}, \ldots, Y_{n}\right]=X_{n}
$$

Remark 2.1

It should be noted that, by conditional expectation property which states that

$E[g(X) \mid Y=y]$ is a function of $y$ for each $g$. For if we have $E|g(X)|<\infty, X_{n}$ is a function of $Y_{1}, \ldots, Y_{n}$ determines the value of $X_{n}$. Also by the law of total probability for expectations,

$$
E\left(X_{n+1}\right)=E\left\{E\left[X_{n+1} \mid Y_{0}, Y_{1}, \ldots, Y_{n}\right]\right\}=E\left(X_{n}\right) \forall n \geq 0 .
$$

And thus by induction,

$$
E\left(X_{n}\right)=E\left(X_{0}\right) \forall n \geq 0
$$

It is useful to think of $Y_{0}, \ldots, Y_{n}$ as information or history up to stage $n$.

Remark 2.2

Equation (2.4) is a matingale property which plays a vital role in the use of optional stopping theorem.

Definition 2.4

Let $\left\{X_{n}\right\}, n \geq 0$ be a discrete time stochastic process, and $\mathrm{F}_{n}$ be the $\sigma-$ algebra generated by $\left\{X_{0}, \ldots, X_{n}\right\}$. A mapping $t: \Omega \rightarrow\{0,1, \ldots, \infty\}$ is called a stopping time with respect to (w.r.t) $\left\{X_{n}\right\}$ ( or w.r.t. $\left\{\mathrm{F}_{n}\right\}$ ) if the event $\{t=n\}$ is completely determined by $\left\{X_{0}, X_{1}, \ldots, X_{n}\right\}$ ( or is a set inf ${ }_{n}$ ).

\subsection{Examples of Stopping Time}

(1) The fixed (that is, constant) $t=k$ is a stopping time.

(2) The first time the process $X_{0}, X_{1}, \ldots$ reaches some subset $A$ of the state space is a stopping time. That is $t=\min \left\{n: X_{n} \in A\right\}$ is a stopping time. This is because

$$
I_{t_{A}=n}\left(X_{0}, \ldots, X_{n}\right)=\left\{\begin{array}{l}
1, \text { if } X_{j} \in A \\
\text { for } j=0, \ldots, n-1 ; X_{n} \in A \\
0, \text { otherwise }
\end{array}\right.
$$

(3) Consider a coin flipping game in which each player plays and win $\$ 100$ or looses with equal probability. We let $Y_{1}, Y_{2}, \ldots$ be independent, and identically distributed random variables, with

$$
P\left[Y_{k}=1\right]=P\left[Y_{k}=-1\right]=\frac{1}{2} .
$$

Let $X_{n}=X_{0}+Y_{1}+\ldots+Y_{n}$ be the player's fortune at stage $n$ of the game. We know that $E\left[X_{n}\right]=X_{0}$. However, let $t=\min \left\{n: Y_{1}+\ldots+Y_{n}\right\}$. Now $[t=n]$ occurs if and only if $Y_{1}+\ldots+Y_{k}<1$ for $k<n$ and $Y_{1}+\ldots .+Y_{n}=1$.

Therefore $t$ is a stopping time.

\section{Martingales Corresponding to Scalars Mean $(\mu)$ and Variance $\left(\sigma^{2}\right)$ of a Random Walk}

Let $Y_{n}, n \geq 1$ be independent and identically distributed random variables and $X_{0}=0$ with probability one. Also, let $X_{n}=Y_{1}+\ldots+Y_{n}$ be $n^{\text {th }}$ partial sum. Denote by $\mu$ and $\sigma^{2}$ the mean and variance of $Y_{1}$, i.e.

$$
\mu=E\left(Y_{1}\right)
$$

and

$$
\sigma^{2}=E\left(Y_{1}-\mu\right)^{2}
$$

Corresponding to these scalar quantities are respectively two martingales $M_{n}, W_{n}$ defined by

$$
M_{n}=X_{n}-n \mu
$$

And 


$$
W_{n}=X_{n}^{2}-n \sigma^{2}
$$

If

$$
X_{n}=\left[\sum_{k=1}^{n}\left(Y_{k}-\mu\right)\right] \text {, }
$$

then

$$
W_{n}=\left[\sum_{k=1}^{n}\left(Y_{k}-\mu\right)\right]^{2}-n \sigma^{2}
$$

\section{Lemma 3.1}

Let $\left\{Y_{n}, n \geq 1\right\}$ be a sequence of independent and identically distributed random variables with mean zero. Let $X_{n}=Y_{1}+\ldots+Y_{n}$. Also, let

$$
M_{n}:=X_{n}-\mu n
$$

Then

$\left\{M_{n}: n \geq 0\right\}$ is a martingale (w.r.t.) $\left\{X_{n}: n \geq 0\right\}$.

\section{Proof}

Given $M_{n}:=X_{n}-\mu n$

$\therefore M_{n+1}=X_{n}+Y_{n+1}-\mu(n+1)$

Taking the expected value and conditioning it on $Y_{0}, \ldots, Y_{n}$, we have

$$
\begin{gathered}
E\left[M_{n+1} \mid Y_{0}, \ldots, Y_{n}\right]=E\left\{\left[X_{n}+Y_{n+1}-\mu(n+1)\right] \mid Y_{0}, \ldots, Y_{n}\right\} \\
=E\left[\left(X_{n} \mid Y_{0}, \ldots, Y_{n}\right)\right]+E\left[Y_{n+1} \mid Y_{0}, \ldots, Y_{n}\right]-\mu(n+1)
\end{gathered}
$$

(by linearity Property of expectation)

$$
\begin{aligned}
& =X_{n}+E\left[Y_{n+1}\right]-\mu n-\mu\left(\text { Since } \mathrm{Y}_{n}, n \geq 1\right. \text { are } \\
& \quad \text { independent }) \\
& =X_{n}-\mu n\left[\text { Since } \mu=E\left(Y_{n}\right)=0\right. \\
& =M_{n}
\end{aligned}
$$

It can $\therefore$ be concluded that $\left\{M_{n}: n \geq 0\right\}$ is a martingale $\mathrm{w}$. r. t. $\left\{X_{n}, n \geq 0\right\}$.

\section{Lemma 3.2}

Let $\left\{Y_{n}, n \geq 1\right\}$ be a sequence of independent and identically distributed random variables with mean $\mu=0$ and finite variance $\sigma^{2}$. Let $X_{n}=Y_{1}+\ldots .+Y_{n}$.

Also, let

$$
W_{n}:=X_{n}^{2}-n \sigma^{2}
$$

Then,

$\left\{W_{n}, n \geq 0\right\}$ is a martingale w.r.t. $\left\{X_{n}, n \geq 0\right\}$.

For the proof see (Ugbebor, Ganiyu and Fakunle (2012)) Lemma 3.3

Let $\left\{Y_{n}, n \geq 1\right\}$ be a sequence of independent and identically distributed random variables with mean $\mu=0$ and finite variance $\sigma^{2}$. Let $X_{n}=Y_{1}+\ldots .+Y_{n}$.

Also, let

$$
M_{n}^{*}:=X_{n}^{2}-n
$$

Then,

$$
\left\{M_{n}^{*}, n \geq 0\right\} \text { is a martingale w. r.t. }\left\{X_{n}, n \geq 0\right\}
$$

Remark 3.1

The proof of lemma 3.3 is similar to the proof for lemma 3.1. The only difference is that in lemma 3.3, a unit variance has been considered. That is

\section{Optional Stopping Theorem [OST]}

The OST as stated and proved by Kannan [1997 ] can be stated as follows.

Let $\left\{X_{n}\right\}, n \geq 0$ be a martingale and $t$ a stopping time. If

(1) $\quad P(t<\infty)=1$

(2) $\quad E\left|X_{t}\right|<\infty$

(3) $\lim _{x \rightarrow \infty} E\left[X_{n} I_{\{t>n\}}\right]=0$

Then,

$$
E\left[X_{t}\right]=E\left[X_{0}\right]
$$

Remark 4.1

'Optional stopping theorem' asserts that a gambler cannot improve his expected gain (fortune) having been given a [finite life time] stopping time (which gives conditions (1) and (2) of the above theorem and a house limit on bets

\{i.e. $\left.\lim _{n \rightarrow \infty}\left[E\left(X_{n} I_{\{t>n\}}\right)\right]=0\right\}$ (which gives condition (3) of the theorem). That is the expected fortune of a gambler with an infinite wealth is zero.

\section{Determination of Mean and Variance of a Stopping Time Using Optional Stopping Theorem (OST)}

\section{Definition 5.2}

A random walk is a mathematical formulation of a trajectory that consists of taking successive random steps,

\section{Definition 5.2}

Let $Y_{0}$ be a fixed positive integer and $\left\{Y_{n}, n \geq 1\right\}$, be the independent and identically distributed jump variables in a random walk $\left\{X_{n}, n \geq 0\right\}$ such that

$$
X_{n}=Y_{0}+Y_{1}+\ldots+Y_{n}
$$

The random walk $\left\{X_{n}, n \geq 0\right\}$ is called simple random walk if 


$$
\left.\begin{array}{c}
p=P\left\{Y_{n}=1\right\} \\
q=P\left\{Y_{n}=-1\right\} \\
\text { and } r=P\left\{Y_{n}=0\right\}
\end{array}\right\}
$$

Where $(p+q+r)=1$ and $0<p, q<1 ; 0 \leq r<1$.

Then

$$
E\left[Y_{n}\right]=n(p-q)
$$

And

$$
\operatorname{Var}\left[Y_{n}\right]=4 n p q
$$

For details of (5.3) and (5.4) see Ganiyu (2006)

\subsection{Statement of the Problem}

Given a random walk of the form

$$
X_{n}:=1+\sum_{k=1}^{n} Y_{k}, n \geq 0, \quad X_{0}=1
$$

with the probability of success $p=\frac{1}{3}$ and probability of failure $q=\frac{2}{3}$ and further that $t$ is a stopping time defined by

$$
t:=\min \left\{n \geq 0: X_{n}=-3\right\} .
$$

The aim here is to determine the mean of the stopping time $t, E(t)$ and the variance of the stopping time $t$, Vart using optional stopping theorem.

\subsection{Solution to the Problem}

Given that $X_{n}:=1+\sum_{k=1}^{n} Y_{k}, n \geq 0$ is a random walk starting at $X_{0}=1$ with $p=\frac{1}{3}$ and $q=\frac{2}{3}$. Assume from (5.5) that $Y_{n}$ are independent and identically distributed random variable. Then for $n=1$, the probability of success and failure are respectively

$$
\left.\begin{array}{c}
p=P\left\{Y_{1}=1\right\} \\
q=P\left\{Y_{1}=-1\right\}
\end{array}\right\}
$$

and $\operatorname{Var}\left[Y_{1}\right]=4 p q=\frac{8}{9}$ by equation (5.4)

The stopping time is defined by

$$
t:=\min \left\{n \geq 0: X_{n}=-3\right\} .
$$

The mean $E(t)$ can be determined as follows.

The martingale corresponding to mean $\mu=E\left[Y_{1}\right]$, a scalar is

$M_{n}=X_{n}-n \mu$, since $\left\{M_{n}, n \geq 0\right\}$ is a martingale w.r.t. $\left\{X_{n}, n \geq 0\right\}$ by Lemma 3.1.

$$
M_{n}=X_{n}-n E\left[Y_{1}\right]=X_{n}-n(p-q)
$$

But for

$$
X_{n}=1+\sum_{n=1}^{n} Y_{k} \text { (given) }
$$

$$
M_{n}=1+\sum_{k=1}^{n} Y_{k}-n(p-q)
$$

Since $\left\{M_{n}, n \geq 0\right\}$ is a martingale w.r.t. $\left\{X_{n}, n \geq 0\right\}$ by Lemma 3.1, then by martingale property and using equation (2.4), this gives

$$
E\left[M_{n}\right]=E\left[M_{0}\right]=E[1]=1
$$

By OST

$$
E\left[M_{t}\right]=E\left[M_{0}\right]=1
$$

But

$$
\begin{aligned}
E\left[M_{t}\right] & =E\left[X_{t}\right]-(p-q) E(t) \text { by equation (5.10) and OST } \\
& =-3-(p-q) E(t)=1\left\{E\left(X_{t}\right)=-3\right\} \text { by equation (5.6) }
\end{aligned}
$$

Solving the last equation, we have

$$
E(t)=\frac{-4}{p-q}=\frac{-4}{\frac{1}{3}-\frac{2}{3}}=12
$$

Therefore, the mean stopping time $E(t)=12$.

The Variance Vart can be determined as follows.

$$
\text { Var } t=E\left(t^{2}\right)-[E(t)]^{2}
$$

The martingale corresponding to the scalar $\sigma^{2}:=E\left(Y_{1}-\mu\right)^{2}$ is given by

$$
\begin{aligned}
W_{n} & =\left[\sum_{k=1}^{n}\left(Y_{k}-\mu\right)\right]^{2}-n \sigma^{2} \\
& \Rightarrow W_{n}=\left[\sum_{k=1}^{n} Y_{k}-n \mu\right]^{2}-n \sigma^{2} \\
& =W\left(X_{n}-1-n \mu\right)^{2}-n \sigma^{2}, \quad\left(\text { for } X_{n}-1=\sum_{k=1}^{n} Y_{k}\right) .
\end{aligned}
$$

$\left\{W_{n}, n \geq 0\right\}$ is a martingale w. r. t. $\left\{X_{n}, n \geq 0\right\}$ (by Lemma 3.2). This leads to 


$$
\begin{gathered}
E\left[W_{n}\right]=E\left[W_{0}\right]=E\left[\left(X_{0}-1-0 . \mu\right)^{2}-0 . \sigma^{2}\right] \\
=E\left[(1-1-0)^{2}-0\right], \text { since } X_{n}=1 \text { by the given definition } \\
\therefore E\left[W_{n}\right]=E\left[W_{0}\right]=0
\end{gathered}
$$

By OST,

$$
\begin{gathered}
E\left[W_{t}\right]=E\left[W_{0}\right]=0 \\
\therefore E\left[W_{t}\right]=E\left[\left(X_{t}-1-t \mu\right)^{2}-t \sigma^{2}\right]=0 \\
=E\left[X_{t}^{2}-2 X_{t}+1-2\left(X_{t}\right) t \mu+2 t \mu+t^{2} \mu^{2}-t \sigma^{2}\right] \\
=E\left[X_{t}^{2}\right]-2 E\left[X_{t}\right]+E[1]-2 E\left[X_{t}\right][E(t)] \mu \\
+2 \mu E(t)+\mu^{2} E\left(t^{2}\right)-\sigma^{2} E[t]=0
\end{gathered}
$$

By equation (5.6)-definition of stopping time

$E\left[X_{t}\right]=-3, E\left[X_{t}^{2}\right]=9$, and by equations (5.8) and (5.12),

$$
\begin{gathered}
\mu=E\left[Y_{1}\right]=-\frac{1}{3} \text { and } E[t]=12 \text { (respectively). } \\
\therefore \sigma^{2}=E\left[Y_{1}-\mu\right]^{2}=E\left[Y_{1}^{2}\right]-2 \mu E\left[Y_{1}\right]+\mu^{2}
\end{gathered}
$$

(by linearity property of expectation)

$$
\begin{aligned}
\therefore \sigma^{2}=E\left[Y_{1}-\mu\right]^{2} & =p+q-2 E\left[Y_{1}\right] E\left[Y_{1}\right]+\left(E\left[Y_{1}\right]\right)^{2} \text { (by equation }(5.2) \\
& =1-2\left(E\left[Y_{1}\right]\right)^{2}+\left(E\left[Y_{1}\right]\right)^{2} \\
& =1-\left(E\left[Y_{1}\right]\right)^{2} \\
& =1-\left(-\frac{1}{3}\right)^{2} \\
& =\frac{8}{9}
\end{aligned}
$$

Now, using equation (5.15)

$$
\begin{aligned}
E\left[W_{t}\right] & =9-2(-3)+1-2(-3)(12)\left(-\frac{1}{3}\right)+2\left(-\frac{1}{3}\right)(12) \\
& +\left(-\frac{1}{3}\right)^{2} E\left[t^{2}\right]-\frac{8}{9}(12)=0 \\
& =-16-\frac{32}{3}+\frac{E\left(t^{2}\right)}{9}
\end{aligned}
$$

$$
E\left(t^{2}\right)=240
$$

The variance of the stopping time $=240-144=96$.

\subsection{The Implication of Martingale Properties in the Use of Optional Stopping Theorem}

In the solution to the problem 3.1, it should be noted that martingale property [used in equations (5.11) and (5.14)] played vital roles in the use of optional stopping theorem for the determination of mean and variance [equations (5.12) and (5.16) respectively]. The implication of

\begin{tabular}{|c|c|c|c|c|}
\hline Stage & Bet & Coin flipping outcome & Time & Gain(loss) \\
\hline 1 & $\$ 100$ & Tail & $t_{1}$ & $-\AA 100$ \\
\hline 2 & $\$ 200$ & Tail & $t_{2}$ & $-\approx 200$ \\
\hline 3 & $\$ 400$ & Tail & $t_{3}$ & $-\AA 400$ \\
\hline 4 & \#800 & Head & $t_{4}$ & $+\$ 800$ \\
\hline
\end{tabular}
martingale property is that the property must be satisfied before the use of optional stopping theorem. For example, this property must be satisfied in using OST 4.0 to prove the impossibility of successful betting strategies for a gambler with a finite lifetime ( which gives condition (i) of OST and a house limit on bets [condition (iii)] of OST).

Suppose that the gambler can wage up to $\# c$ on a fair coin flipping game at times $t_{1}, t_{2}, t_{3}, \ldots$, winning his wager if the coin comes up heads and losing it if the coin comes up tails. Suppose further that he can quit whenever he likes, but cannot predict the outcome of the gambles that have not happened yet. Then, the gambler's fortune over time is a martingale and the time $t$ at which he decides to quit (or goes broke and is forced to quite) is a stopping time. So the OST says that $E\left[X_{t}\right]=E\left[X_{0}\right]$. What this implies is that the gambler leaves with the same amount of money on the average as when he started. The martingale property can easily be seen in the following table of betting strategy.

\subsection{Analysis of the Betting System}

Assume that the gambler is playing a con flipping game and started with $\$ 100$ at stage 1, he loses the $\$ 100$ at time $t_{1}$ with the appearance of tail. At stage 2, he releases $\$ 200$ (double his bet on stage 1), he loses $\$ 200$ at time $t_{2}$ with the appearance of tail. At stage 3, he releases $\$ 400$ (double his bet on stage 2), he loses $\$ 400$ at time $t_{3}$ with the appearance of tail. At stage 4, he releases $\$ 800$ (double his bet on stage 3 ), he now gain $\$ 800$ at time $t_{4}$ with the appearance of head. The stopping time is $t_{4}$. The total gain (loss) of the gamble is $-\$ 100-\$ 200-\$ 400+\$ 800=$ $\$ 100$.

At stage 4 , the gambler is now forced back to stage 1 by gaining a sum of $\$ 100$. This implies that the gambler had to leave with the same amount of money as when he started. Therefore, martingale property is satisfied.

\section{Conclusion}

This paper showed the dependency of optional stopping theorem on martingale property before the application of optional stopping theorem. It has given an intuitive meaning of optional stopping theorem telling us that even with a well-chosen strategy for stopping a game, under reasonable hypotheses, a martingale is a fair game. To establish the dependency of martingale property on 
optional stopping theorem, optional stopping theorem was applied to determine the mean and variance of a stopping. A simple example relating to betting system of a gambler with limited amount of money was also provided. The analysis of the betting system showed that the gambler leaves with the same amount of money as when he started and therefore satisfied martingale property. It can therefore be concluded that martingale property is a dependable tool in the use of optional stopping theorem (OST).

\section{References}

[1] Ganiyu A. A. (2006). Theoretical Framework of Martingales Associated with Random Walk for Foreign Exchange Rate Determination. An unpublished M.Phil. dissertation, University of Ibadan, Ibadan. Pp 7,11,14,20-21,71-74

[2] Hazewinkel M. (2001). "Martingale" (http://www.encyclopediaofmaths.org?/index.php?title $=\mathrm{p} / \mathrm{m}$ 062570, Encyclopedia of mathematics, Springer, ISBN 9781-55608-010-4.

[3] Kannan, D. (1997). "An introduction to Stochastic Process". North Holland Series in Probability and Applied Mathematics. P. 196, (222-223), 24-25, 28-29.

[4] Karlin S. and H. Taylor (1975). A first course in Stochastic Processes. Second Edition. Academic Press, Section 6.3, P. (253-262).
[5] Shiu, E.S.W and Gerber H.U. (1994a). "Option Pricing by Esscher Transforms," . Transactions, Society of Actuaries XLVI:99-140; Discussion 141-191.

[6] Shiu, E.S.W. and Gerber H.U. (1994b). "Martingales Approach to Pricing Perpectual American Option,". ASTIN Bulletin 24:195-220.

[7] Shiu, E.S.W. and Gerber H.U. (1996a). "Martingales Approach to Perpectual American Options on Two Stocks, ". Mathematical Finance 6:303-322.

[8] Shiu, E.S.W. and Gerber H.U. (1996b). "Actuarial Bridges to Dynamic Hedging and Option Pricing," Insurance: Mathematics and Economics 18:183-218. Walk for Foreign Exchange Rate Determination. An unpublished M.Phil. dissertation, University of Ibadan, Ibadan. Pp 7,11,14,2021,71-74

[9] Ugbebor O.O. and Ganiyu A.A. (2007). Martingales Associated with Random Walk Model for Foreign Exchange Rate Determination. Nigerian Mathematical Society Journal, Vol. 26 Pp (19-31).

[10] [10 ] Ugbebor O.O., Ganiyu A.A. and Fakunle I. (2012). "Optional Stopping Theorem as an Indispensable Tool in the Determination of Ruin Probability and Expected Duration of a Game". Journal of the Nigerian Association of Mathematical Physics", Vol. 21, Pp 85-93.

[11] http://nvis.neurodebates.com/on/Optional_stopping_theorem 